\title{
A study of the Martian water vapor over Hellas using OMEGA and PFS aboard Mars Express
}

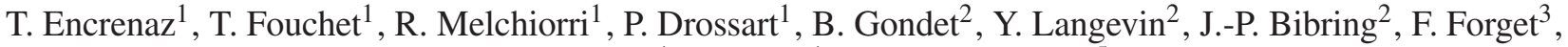 \\ L. Maltagliati ${ }^{4}$, D. Titov ${ }^{4}$, and V. Formisano ${ }^{5}$ \\ 1 LESIA, Observatoire de Paris, CNRS, UPMC, Univ. Denis Diderot, 92195 Meudon, France \\ e-mail: therese.encrenaz@obspm.fr \\ 2 Institut d'Astrophysique Spatiale, Orsay Campus, 91405 Orsay, France \\ IPSL/LMD, place Jussieu, 75231 Paris Cedex 05, France \\ 4 MPS, Max-Planck St. 2, 37191 Katlenburg-Lindau, Germany \\ 5 INAF-IFSI, Via del Fosso del Cavaliere 100, 00133 Rome, Italy \\ Received 20 December 2007 / Accepted 22 February 2008
}

\section{ABSTRACT}

\begin{abstract}
We used the OMEGA imaging spectrometer aboard Mars Express to study the evolution of the water vapor abundance over the Hellas basin, as a function of the seasonal cycle. The $\mathrm{H}_{2} \mathrm{O}$ column density is found to range from very low values (between southern fall and winter) up to more than $15 \mathrm{pr}-\mu \mathrm{m}$ during southern spring and summer. The general behavior is consistent with the expected seasonal cycle of water vapor on Mars, as previously observed by TES and modeled. In particular, the maximum water vapor content is observed around the southern solstice, and is significantly less than its northern couterpart. However, there is a noticeable discrepancy around the northern spring equinox $\left(L_{\mathrm{s}}=330-60^{\circ}\right)$, where the observed $\mathrm{H}_{2} \mathrm{O}$ column densities are significantly lower than the values predicted by the GCM. Our data show an abrupt enhancement of the water vapor column density (from 3 to 16 pr- $\mu \mathrm{m}$ ) on a timescale of 3 days, for $L_{\mathrm{s}}=251-254^{\circ}$. Such an increase, not predicted by the GCM, was also occasionally observed by TES over Hellas during previous martian years at the same season; however, its origin remains to be understood.
\end{abstract}

Key words. planets and satellites: individual: Mars - infrared: solar system

\section{Introduction}

The water vapor cycle of Mars has been extensively studied from space, both in the solar reflected spectrum and in the thermal regime. IRIS aboard Mariner 9 used the thermal infrared range (Conrath et al. 1973), while the MAWD experiment aboard the Viking spacecraft (Farmer et al. 1977; Jakosky \& Haberle 1992; Fedorova et al. 2004) used the $\mathrm{H}_{2} \mathrm{O} 1.38-\mu \mathrm{m}$ band in the reflected part of the spectrum. This band was also used for a ground-based monitoring at high spectral resolution (Sprague et al. 1996, 2003). The TES thermal instrument aboard Mars Global Surveyor, working in the middle-infrared range, provided the latest reference database for a complete map of water vapor on Mars as a function of latitude and longitude over the three consecutive seasonal cycles (Smith 2002, 2004).

More recently, water vapor has been studied with three instruments of the Mars Express orbiter, SPICAM, OMEGA, and PFS. The PFS long-wavelength data were used in the thermal regime (Fouchet et al. 2007), while both the OMEGA and SPICAM analyses were based on the reflected part of the spectrum. The OMEGA analysis used the $\mathrm{H}_{2} \mathrm{O}$ 2.6- $\mu \mathrm{m}$ band (Encrenaz et al. 2005; Melchiorri et al. 2007a; Maltagliati et al. 2007), and the SPICAM results were obtained from the $1.38-\mu \mathrm{m}$ water band (Fedorova et al. 2007). As compared with the thermal regime, this method applied in the near-infrared range has the advantage of showing, to first order, little dependence upon the thermal properties of the atmosphere. In contrast, it is more sensitive to scattering effects that must be taken into account. Overall, the Mars Express instruments have measured lower values than the Viking/MAWD measurements and than the earliest
MGS/TES results (Smith 2004), but they do agree with the revised TES database (Smith, priv. comm.; Fouchet et al. 2007) and with ground-based measurements (Sprague et al. 2003).

In the present study, we focus on the evolution of the watervapor column density above Hellas basin over a seasonal cycle. Our work aims at comparing observations and modelization of the water vapour amount in this region of complex topography. Two instruments aboard Mars Express have been used, OMEGA, and PFS/LW. The OMEGA analysis is a continuation of a study performed at $2.6 \mu \mathrm{m}$ (Melchiorri et al. 2007a), and also a continuation of a study performed over the Hellas basin on the (2-0) band of $\mathrm{CO}$ at $2.35 \mu \mathrm{m}$ (Encrenaz et al. 2006), which has shown evidence of a significant $\mathrm{CO}$ enhancement during southern winter, in full agreement with theoretical predictions (Forget et al. 2006). In addition, because of the high surface pressure of this area, the water-vapor band depths are usually stronger and can be measured with better accuracy than elsewhere on the Martian disk, and reliable information can be retrieved for each individual orbit by integrating the spectra over the region of lowest altitude. The PFS work is based on the analysis of the $\mathrm{H}_{2} \mathrm{O}$ rotational lines in the thermal regime (Fouchet et al. 2007). Observations and data analysis are presented in Sect. 2. Radiative transfer modeling is described in Sect. 3. Results are presented and discussed in Sect. 4.

\section{Observations and data analysis}

\subsection{OMEGA}

Since January 2004, the OMEGA imaging spectrometer (Bibring et al. 2004) aboard Mars Express operates from $0.3 \mu \mathrm{m}$ 


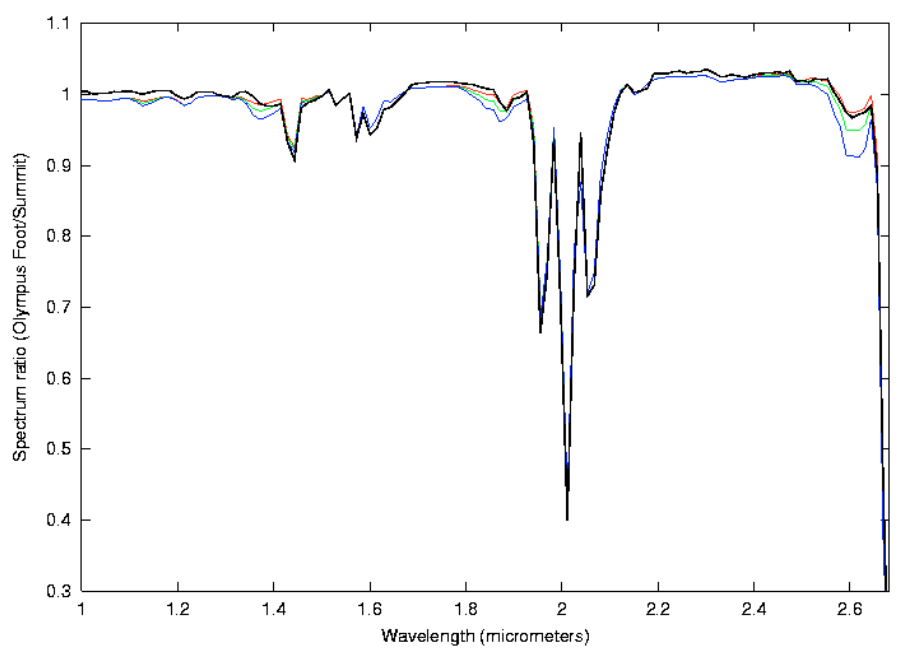

Fig. 1. Black curve: ratio of two averaged OMEGA spectra (each integrated over 352 individual spectra), recorded at the foot (30N, 227E) and at the summit $(17 \mathrm{~N}, 227 \mathrm{E})$ of Olympus Mons respectively (Orbit 37-3), between 1.0 and $2.65 \mu \mathrm{m}$. Color curves: synthetic ratios of spectra (Ps=1.1 mbar at the summit, 7.5 mbar at the foot). The CO mixing ratio is $800 \mathrm{ppm}$. From top to bottom: $Q\left(\mathrm{H}_{2} \mathrm{O}\right)=150 \mathrm{ppm}$ (red), $300 \mathrm{ppm}$ (green), and $600 \mathrm{ppm}$ (blue). The best fit is obtained for $Q\left(\mathrm{H}_{2} \mathrm{O}\right)=150 \mathrm{ppm}$. This figure is taken from Encrenaz et al. (2005).

to $5.2 \mu \mathrm{m}$, with a spectral sampling of $14 \mathrm{~nm}$ in the $1.0-2.7 \mu \mathrm{m}$ range. The instantaneous field of view (IFOV) is $1.2 \mathrm{mrad}$, which corresponds to about $300 \mathrm{~m}$ at the martian surface near periapsis. Its high spatial resolution, combined with a moderate spectral resolving power, makes OMEGA ideal for a mineralogic mapping of the martian surface, but the instrument has also demonstrated its capability to map minor atmospheric constituents.

The 1.0-2.7 $\mu \mathrm{m}$ spectrum of Mars (Fig. 1) is dominated by signatures of $\mathrm{CO}_{2}$, in particular two strong bands at $2.7 \mu \mathrm{m}$ and $2.0 \mu \mathrm{m}$. Water shows three clear bands at $1.38 \mu \mathrm{m}, 1.9 \mu \mathrm{m}$, and $2.6 \mu \mathrm{m}$. This last band, which is by far the strongest one, was used to monitor the water-vapor column density (Encrenaz et al. 2005; Melchiorri et al. 2007a). A weak band of CO is present at $2.35 \mu \mathrm{m}$, which was used to study the $\mathrm{CO}$ seasonal cycle over Hellas by Encrenaz et al. (2006).

In all cases, we divided each individual spectrum by the averaged spectrum of the Olympus summit, taken as a reference, and we followed the same procedure in the present study. The main reason for using this procedure is to eliminate the uncertainty associated to the OMEGA transfer function within the $\mathrm{H}_{2} \mathrm{O}$ band, for which the absolute accuracy cannot be better than a percent. As the $\mathrm{H}_{2} \mathrm{O}$ band depth over the summit of Olympus Mons is less than 2 percent, the calibration uncertainty induces a strong uncertainty (more than 50 percent) on the measured $\mathrm{H}_{2} \mathrm{O}$ column density over the Olympus summit. By dividing all OMEGA spectra by the spectrum of the Olympus summit, we remove this uncertainty. However, by doing so, we need to assume that the $\mathrm{H}_{2} \mathrm{O}$ mixing ratio is constant between the top and the foot of Olympus Mons, which is also a debatable assumption, as discussed below.

Figure 1 shows the retrieval of the $\mathrm{H}_{2} \mathrm{O}$ mixing ratio in the vicinity of Olympus Mons, using the above technique. As explained in more detail in our previous studies, a mean spectrum recorded along Orbit 37-7, at a mean latitude of $20^{\circ} \mathrm{N}$, was divided by our reference spectrum, taken at the summit of Olympus Mons. The surface pressure of the reference spectrum was inferred from the depth of the $\mathrm{CO}_{2}$ bands over
Table 1. Summary of OMEGA observations over Hellas.

\begin{tabular}{lcccc}
\hline \hline Orbit & $L_{\mathrm{s}}$ & $P_{\mathrm{s}}$ (mbar) & Airmass & Local time \\
\hline $30-1$ & 335.7 & 12.5 & 1.17 & $14: 00$ \\
$41-3$ & 337.9 & 11.5 & 1.20 & $13: 00$ \\
$47-1$ & 339.0 & 11.5 & 1.20 & $13: 00$ \\
$284-0$ & 16.6 & 11.5 & 1.15 & $11: 00$ \\
$422-1$ & 36.1 & 10.5 & 1.75 & $09: 00$ \\
$521-1$ & 48.5 & 11.0 & 2.25 & $09: 00$ \\
$554-1$ & 52.6 & 11.2 & 2.70 & $08: 00$ \\
$1186-4$ & 132.0 & 12.5 & 1.52 & $14: 00$ \\
$1241-5$ & 139.5 & 12.5 & 1.52 & $13: 00$ \\
$1935-4$ & 251.7 & 11.6 & 1.55 & $17: 00$ \\
$1946-4$ & 253.6 & 11.5 & 1.47 & $17: 00$ \\
$2177-4$ & 294.0 & 11.6 & 1.55 & $17: 00$ \\
\hline
\end{tabular}

the $1.0-2.7 \mu \mathrm{m}$ spectral range. The inferred water vapor mixing ratio (assumed to be constant at the top and the foot of Olympus Mons) is $150 \mathrm{ppm}$, which corresponds to a column density of $1.6 \mathrm{pr}-\mu \mathrm{m}$ above the summit of Olympus Mons. As mentioned above, there is some uncertainty associated with the assumption of a constant $\mathrm{H}_{2} \mathrm{O}$ mixing ratio over the volcanoes. Mars Express instruments disagree whether the watervapor mixing ratio stays constant from the bottom to the top of the volcano. Using PFS/LW data, Fouchet et al. (2007) found the $\mathrm{H}_{2} \mathrm{O}$ mixing ratio to be constant within a factor 2 over Olympus Mons, while Maltagliati et al. (2006, 2008) used OMEGA data to find some spatial and temporal variabilities with, in most of the cases, a local enhancement of the water mixing ratio by a factor of 4 around the summits. Assuming a conservative maximum possible enhancement by a factor of 4 in the mixing ratio over Olympus Mons, the resulting water-vapor column density would be $6.4 \mathrm{pr}-\mu \mathrm{m}$ instead of $1.6 \mathrm{pr}-\mu \mathrm{m}$. Indeed, a value of 5$6 \mathrm{pr}-\mu \mathrm{m}$ is measured by Maltagliati et al. (2008) on Orbit 37 at $20^{\circ} \mathrm{N}$ latitude (for the same orbit, however, PFS data give a value of $2+/-1$ pr- $\mu \mathrm{m}$; Fouchet et al. 2007). We thus conclude that our method can generate an uncertainty of about $5 \mathrm{pr}-\mu \mathrm{m}$.

To study the temporal variation in the $\mathrm{H}_{2} \mathrm{O}$ column density over the Hellas basin, we considered all orbits crossing Hellas from the first one (Orbit 30, 2004 Jan. 18, $L_{\mathrm{s}}=336^{\circ}$, MY 26) to Orbit 2177 (2005 Sep. 24, $L_{\mathrm{s}}=294^{\circ}$, MY 27). We first selected the orbits from the latitude-longtitude criteria, then, within each orbit, we selected the spectra corresponding to the plateau at constant maximum surface pressure, extracted from the European martian climate database (Forget et al. 1999). We removed the orbits that were contaminated by bad pixels or by a strong mineralogic or water ice signature. For orbits recorded after Orbit 2177, data are unfortunately contaminated by bad pixels which make the retrieval of the continuum much more hazardous. For this reason we kept the original selection used for our $\mathrm{CO}$ analysis, which covers a full seasonal cycle. As in the case of our $\mathrm{CO}$ analysis, we used two references (both corresponding to Olympus summit), depending on the integrating time of the data. Spectra recorded with $2.5 \mathrm{~ms}$ integrating times (orbits 1935 and above) have been divided by the reference of Orbit 37-3, also recorded at $2.5 \mathrm{~ms}$. For data recorded at $5 \mathrm{~ms}$ (orbits 30 to 1241), we have used a reference taken at Orbit 501-4, also registered with $5 \mathrm{~ms}$ integration time. Table 1 summarizes the observations. 


\section{2. $P F S / L W$}

The Planetary Fourier Spectrometer is an infrared doublependulum interferometer working in two different channels. The short wavelength channel (SWC) covers wavenumbers from 1700 to $8200 \mathrm{~cm}^{-1}(1.2-5.9 \mu \mathrm{m})$ while the long wavelength channel (LWC) operates in the range $250-1700 \mathrm{~cm}^{-1}$ $(5.9-40 \mu \mathrm{m})$. In principle, the availability of the two channels makes it possible to measure water in both the thermal and solar reflected components simultaneously. In the present analysis we have used data from the LWC. Its detector is a pyroelectric $\left(\mathrm{LiTaO}_{3}\right)$ working at a temperature of $287 \mathrm{~K}$. The LWC is characterized by an unapodized spectral resolution of $1.3 \mathrm{~cm}^{-1}$, a sampling step of $1.0 \mathrm{~cm}^{-1}$, and a field of view of $50 \mathrm{mrad}$ (FWHM). This corresponds to $12 \mathrm{~km}$ on Mars surface when viewed from periapsis. A more detailed description of the PFS instrument can be found in Formisano et al. (2005).

The PFS LW channel calibration has been presented in detail by Giuranna et al. (2005). The instrument spectral response function was obtained both from laboratory measurements and from flight measurements. Laboratory measurements were obtained by looking at a blackbody tunable within a wide range of temperatures (170-270 K). In-flight radiometric calibration measurements were taken by looking at the internal blackbody and pointing the scanner towards deep space. The response functions derived from laboratory and in-flight measurements are in excellent agreement. One difficulty in the calibration process arises from the evolution of the optics temperature during an observation session (the detector temperature remains stable within $\pm 0.05 \mathrm{~K}$ ). This evolution is measured by looking at deep space at the beginning and at the end of a session, and then taken into account in the calibration process. Within the wavenumber range of interest for this study $\left(250-850 \mathrm{~cm}^{-1}\right)$, the noise equivalent spectral radiance (NESR) of a single spectrum varies between $7 \times 10^{-8}$ and $1.5 \times 10^{-7} \mathrm{~W} \mathrm{~cm}^{-2} \mathrm{sr}^{-1} / \mathrm{cm}^{-1}$ (corresponding to a signal-to-noise ratio of $50-100$ on a mid-latitude daytime spectrum).

\section{Radiative transfer modeling}

\subsection{OMEGA}

We calculated the absorption due to the martian atmospheric gases over the 1.0-2.7 $\mu \mathrm{m}$ range, using the line-by-line radiative transfer code described in our earlier studies, which includes the spectral signatures of $\mathrm{CO}_{2}, \mathrm{H}_{2} \mathrm{O}$, and $\mathrm{CO}$ (Encrenaz et al. 2005, 2006; Melchiorri et al. 2007a). Scattering effects are not taken into account in this code and are evaluated following the method described for the $\mathrm{CO}$ analysis (see below). The surface pressure and the mean temperature profile over Hellas are taken from the global climate model (GCM) developed at the LMD and at Oxford (European martian climate database, Forget et al. 1999; Lewis et al. 1999). For each observation listed in Table 1, we calculated a set of synthetic spectra of the $\mathrm{H}_{2} \mathrm{O}$ band at $2.6 \mu \mathrm{m}$, corresponding to its surface pressure and airmass, for water-vapor mixing ratios of $0,50,100,300$, and $500 \mathrm{ppm}$. The inferred $\mathrm{H}_{2} \mathrm{O}$ mixing ratios, with the corresponding column densities, were obtained from a least-square procedure. In order to account for the effect of aerosol scattering, we used the method described in our $\mathrm{CO}$ analysis. We generated synthetic models of the 2.6- $\mu \mathrm{m}$ band of $\mathrm{H}_{2} \mathrm{O}$ including dust multiple scattering, using a two-stream radiative transfer code. Following Ockert-Bell et al. (1997), we assumed as dust scattering properties $\omega_{0}=0.95$ for the single scattering albedo and $g=0.67$ for the asymmetry factor of the phase function. Our analysis shows that, for a

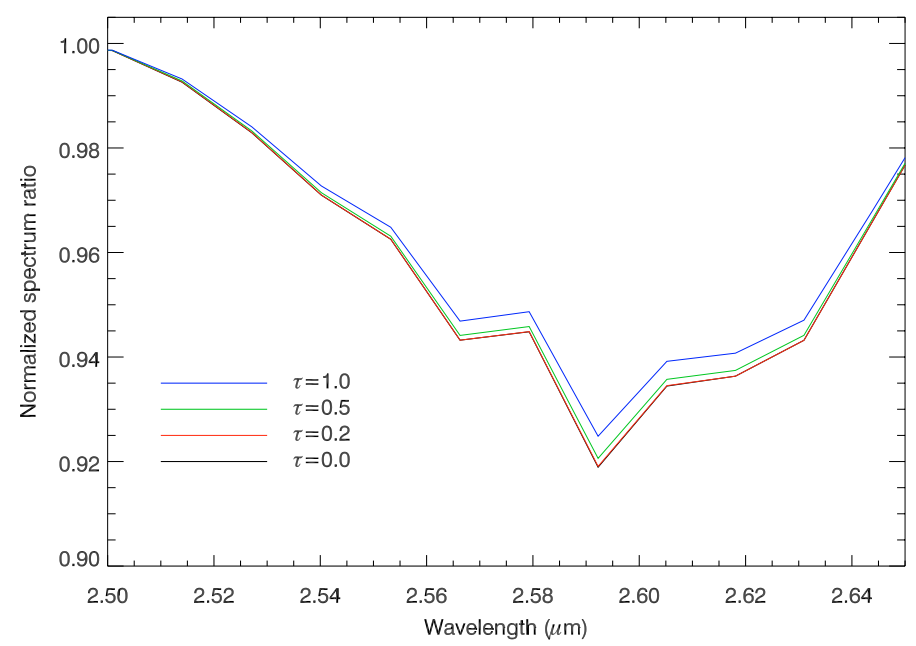

Fig. 2. Effect of dust scattering on the depth of the $\mathrm{H}_{2} \mathrm{O}$ band at $2.6 \mu \mathrm{m}$. The surface pressure is 10 mbars, the airmass is 1.3 , and the $\mathrm{H}_{2} \mathrm{O}$ mixing ratio $150 \mathrm{ppm}$. Dust opacity, from top to bottom: 1.0, 0.5, 0.2, 0.0 .

broad range of water-vapor mixing ratios, the effect of aerosol scattering lowers the band depth by about 2 and 8 percent for dust optical depths of 0.5 and 1.0, respectively. An example of this effect is shown in Fig. 2, corresponding to an $\mathrm{H}_{2} \mathrm{O}$ mixing ratio of $150 \mathrm{ppm}$. For each orbit, we applied the associated correction by estimating the dust content from the GCM and simultaneously checking this value by fitting the depth of the $\mathrm{CO}_{2}$ $2.0-\mu \mathrm{m}$ band. In order to estimate the error bars, we took two extreme cases, the zero dust model and the GCM dusty model. The same method was applied in our CO analysis (Encrenaz et al. 2006).

\subsection{PFS/LW}

The water-vapor retrieval method from PFS/LW is fully described in Fouchet et al. (2007). The forward radiative transfer modeling of PFS/LW is carried out using a line-by-line model including only the gaseous absorptions by $\mathrm{CO}_{2}$ and $\mathrm{H}_{2} \mathrm{O}$. The model assumes a plane-parallel geometry and does not take dust scattering into account. The spectroscopic parameters are taken from the GEISA database (Jacquinet-Husson et al. 1999), except for the $\mathrm{CO}_{2}$-broadening parameters. For water, we use the parameters calculated by Gamache et al. (1995). The temperature profile is retrieved first. The inversion of a temperature profile from planetary thermal emission is a typically ill-posed problem where arbitrarily small changes in the observed radiances lead to large differences in the inverted profile, but it is possible to retrieve a meaningful temperature profile with the regularization and stabilization methods. In the case of the water column-density retrieval, the best-fit model is estimated through the usual $\chi^{2}$ cost function across the $305-505 \mathrm{~cm}^{-1}$ interval. The NESR translates into an uncertainty of $1-2 \mathrm{pr}-\mu \mathrm{m}$ on the retrieved water-column abundance.

\section{Results and discussion}

Table 2 summarizes the water-vapor column densities inferred from our data. while Figs. 3, 4 show examples of spectra in the 2.6- $\mu \mathrm{m}$ water vapor band and the rotational band for different $L_{\mathrm{s}}$. It can be seen that the water vapor content measured by both OMEGA and PFS/LW remains very small for $L_{\mathrm{s}}$ between $330^{\circ}$ and $150^{\circ}$. Then OMEGA observes a very abrupt increase on a 

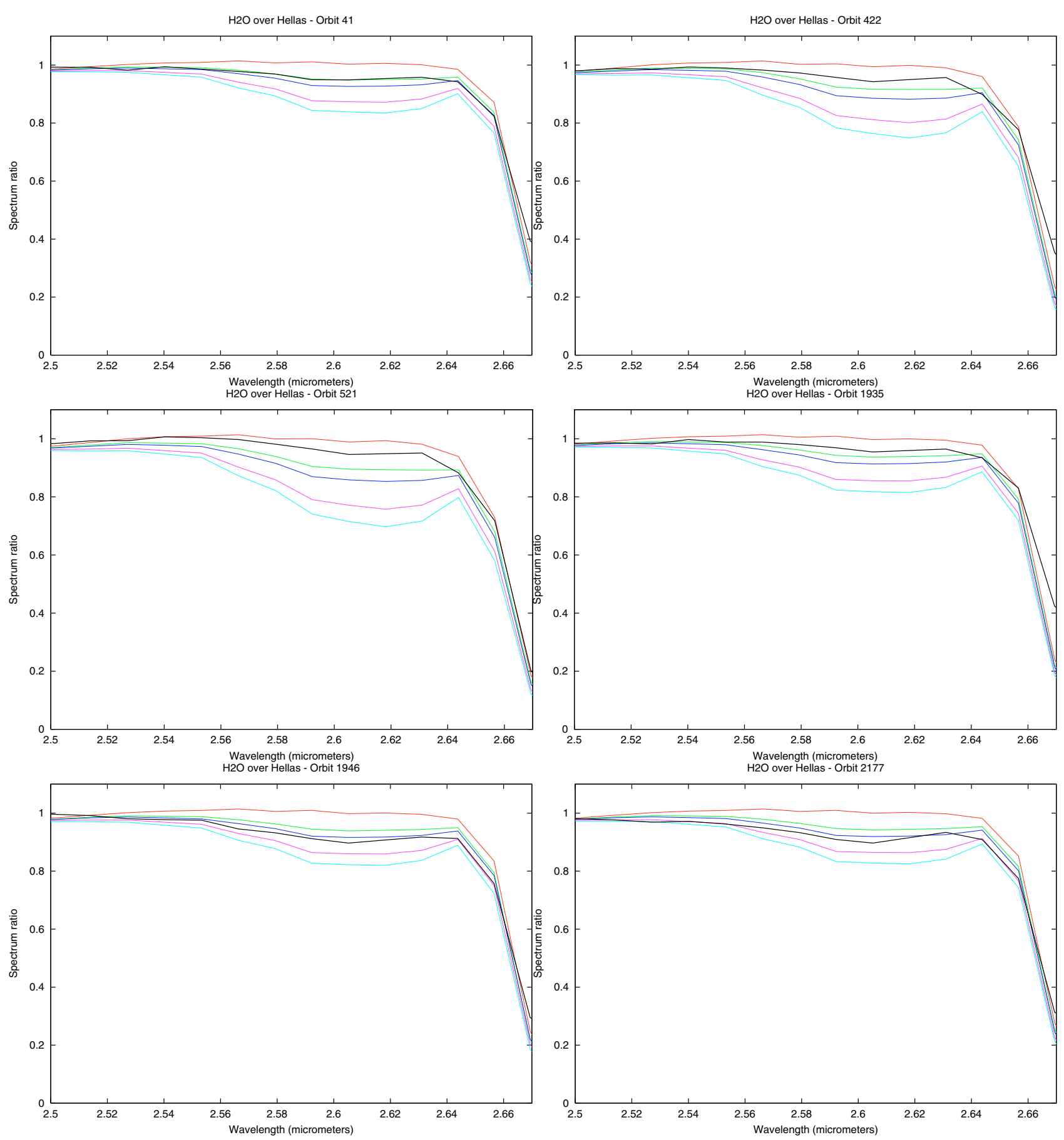

Fig. 3. Comparison between the averaged OMEGA spectra over Hellas (black curve) divided by the reference spectrum taken at the summit of Olympus Mons to synthetic ratio of spectra (color lines), calculated for the surface pressure and the airmass of the observation, with, from top to bottom: $\left[\mathrm{H}_{2} \mathrm{O}\right]=0,50,100,300,500 \mathrm{ppm}$. Upper left panel: $L_{\mathrm{s}}=337.9^{\circ}$ (orbit 41-3); upper right panel: $L_{\mathrm{s}}=36.1^{\circ}$ (orbit $422-1$ ); middle left panel: $L_{\mathrm{s}}=48.5^{\circ}$ (orbit 521-1); middle right panel: $L_{\mathrm{s}}=251.7^{\circ}$ (orbit 1935-4); lower left panel: $L_{\mathrm{s}}=253.6^{\circ}$ (orbit 1946-4); lower right panel: $L_{\mathrm{s}}=295.9^{\circ}$ (orbit 2177-4).

timescale of 3 days, between $L_{\mathrm{s}}=252^{\circ}$ (Orbit 1935) and $L_{\mathrm{s}}=$ $254^{\circ}$ (Orbit 1946), as shown in Fig. 3. This difference appears to be real, as illustrated in Fig. 5, which shows that the water band in the Orbit-1946 data is clearly stronger than in the data of Orbit 1935. For comparison, the spectra recorded south of Hellas during the same orbits do not show any increase in the watervapor content. The high water content observed over Hellas on Orbit 1946 is also observed later for $L_{\mathrm{S}}=294^{\circ}$ (Orbit 2177, see Fig. 3). Unfortunately, PFS was switched off during this period.
For each OMEGA spectrum, the water-vapor mixing ratio retrieved from the data has been corrected for the effect of aerosol scattering and converted into the water vapor column density (Table 2). Figure 10 shows the evolution of the observed $\mathrm{H}_{2} \mathrm{O}$ column density as a function of $L_{\mathrm{s}}$. The OMEGA error bars do not include the systematic uncertainty of $5 \mathrm{pr}-\mu \mathrm{m}$ associated to the measurement of $\mathrm{H}_{2} \mathrm{O}$ over Olympus Mons (Sect. 2). The agreement between the OMEGA data and and the model would be improved if the OMEGA results were shifted upward 
Table 2. Water vapor measurements over Hellas.

\begin{tabular}{lccc}
\hline \hline Orbit & $L_{\mathrm{s}}$ & \multicolumn{2}{c}{$\mathrm{H}_{2}$ O column density $(\mathrm{pr}-\mu \mathrm{m})$} \\
& & OMEGA & PFS/LW \\
\hline $30-1$ & 335.7 & $8.4 \pm 3.0$ & $7.6 \pm 1.3$ \\
$41-3$ & 337.9 & $6.3 \pm 2.3$ & $6.0 \pm 1.0$ \\
$47-1$ & 339.0 & $6.3 \pm 2.3$ & $\mathrm{NA}$ \\
$284-0$ & 16.6 & $6.0 \pm 3.0$ & $4.2 \pm 1.2$ \\
$422-1$ & 36.1 & $4.0 \pm 3.0$ & $<10$ \\
$521-1$ & 48.5 & $0.0(+4.0,-0.0)$ & $<10$ \\
$554-1$ & 52.6 & $0.0(+4.0,-0.0)$ & $<10$ \\
$1186-4$ & 132.0 & $0.0(+4.0,-0.0)$ & $<10$ \\
$1241-5$ & 139.5 & $2.7 \pm 2.7$ & $8 \pm 1.5^{1}$ \\
$1935-4$ & 251.7 & $2.7 \pm 2.7$ & $\mathrm{NA}$ \\
$1946-4$ & 253.6 & $16.5 \pm 3.0$ & $\mathrm{NA}$ \\
$2177-4$ & 295.9 & $16.0 \pm 3.0$ & $\mathrm{NA}$ \\
\hline
\end{tabular}

${ }^{1}$ In the North part of Hellas only. The column density drops below $4 \mathrm{pr}-\mu \mathrm{m}$ in the South of the crater.

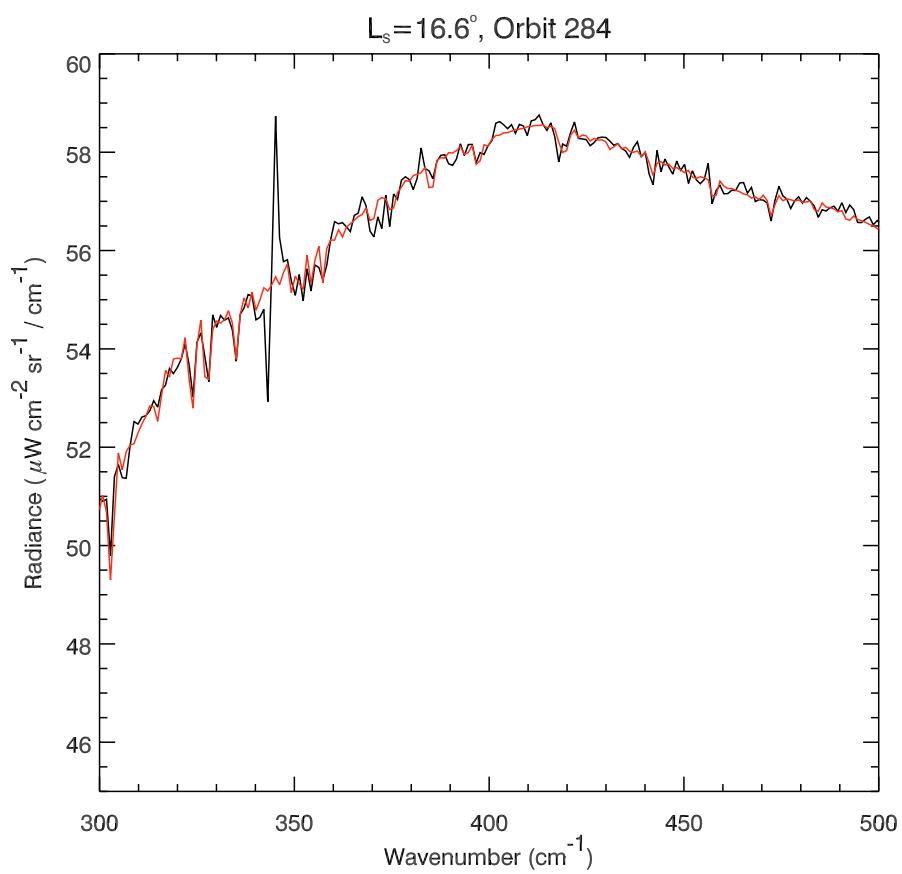

Fig. 4. Comparison between the averaged PFS spectrum over Hellas (black curve) obtained during orbit 284 to the best-fit synthetic spectrum (red line). The spike present in the PFS/LW spectrum at $\sim 345 \mathrm{~cm}^{-1}$ is caused by mechanical vibrations aboard the spacecraft.

by $5 \mathrm{pr}-\mu \mathrm{m}$; however, the agreement between the OMEGA and PFS results would be degraded.

\subsection{Comparison with the GCM}

Figure 6 shows a comparison of the OMEGA and PFS retrieval with the expected evolution as modeled by the GCM. First, very good agreement is observed between the OMEGA and the PFS data points. There is also good agreement with the GCM during the southern summer $\left(L_{\mathrm{s}}=250-300^{\circ}\right)$ when the watervapor content is at its maximum, and, more marginally, during the northern summer $\left(L_{\mathrm{s}}=30-150^{\circ}\right)$ where the observed $\mathrm{H}_{2} \mathrm{O}$ column density tends to be lower than the predictions. In contrast, there is a significant discrepancy around the northern

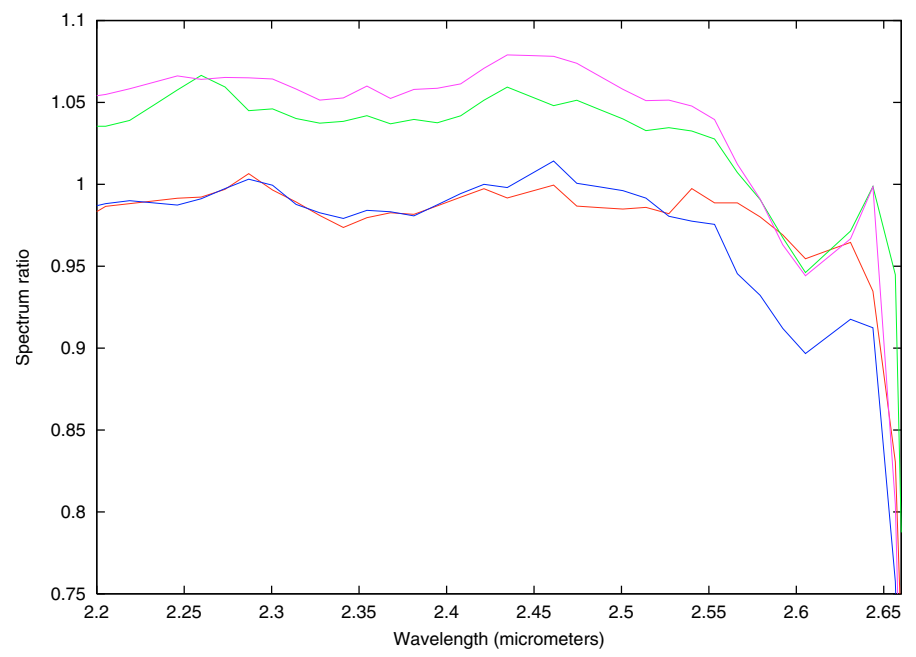

Fig. 5. The averaged observed spectrum over Hellas divided by the reference spectrum taken at the summit of Olympus Mons, for two successive orbits: red, Orbit 1935-4 and blue, Orbit 1946-4 (Fig. 3). The averaged spectra taken south of Hellas (48S-64S) are also shown for comparison (red: Orbit 1935; pink: Orbit 1946).

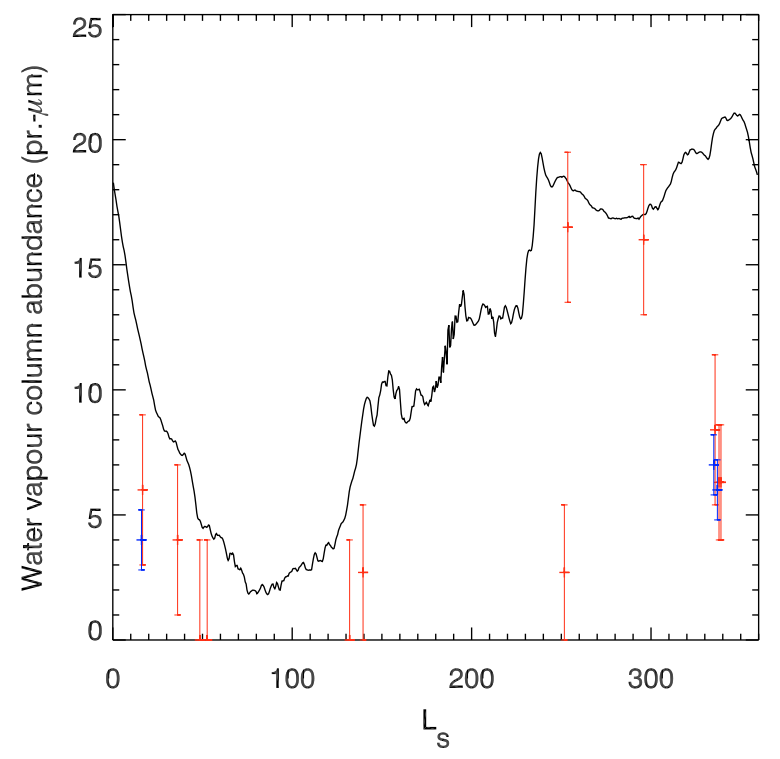

Fig. 6. The water-vapor column abundance measured by OMEGA (red) and PFS/LW (blue) over Hellas as a function of the solar longitude. The GCM predictions are shown for comparison (black curve).

spring equinox $\left(L_{\mathrm{s}}=330-30^{\circ}\right)$ for which low abundances are measured, while high values are expected from the GCM.

One could wonder whether the topography of the Hellas basin might induce local variations. According to the GCM, this should not be the case: Fig. 7 shows the expected distribution of the water column density, normalized to the $610 \mathrm{~Pa}$ reference pressure level, as a function of latitude and longitude near the equinox $\left(L_{\mathrm{s}}=300-330^{\circ}\right)$. It can be seen that no longitude variations are expected (note that the uncorrected $\mathrm{H}_{2} \mathrm{O}$ column density map would show a strong increase within the Hellas basin, as the simple result of the high surface pressure).

Another parameter to take into account is the local time. Indeed, a recent study by Melchiorri et al. (2007b) found evidence of an increase in the water-vapor content between the morning and the evening, at high southern latitudes, for $L_{\mathrm{s}}$ values close to $260^{\circ}$ (southern summer). Table 1 , however, shows 


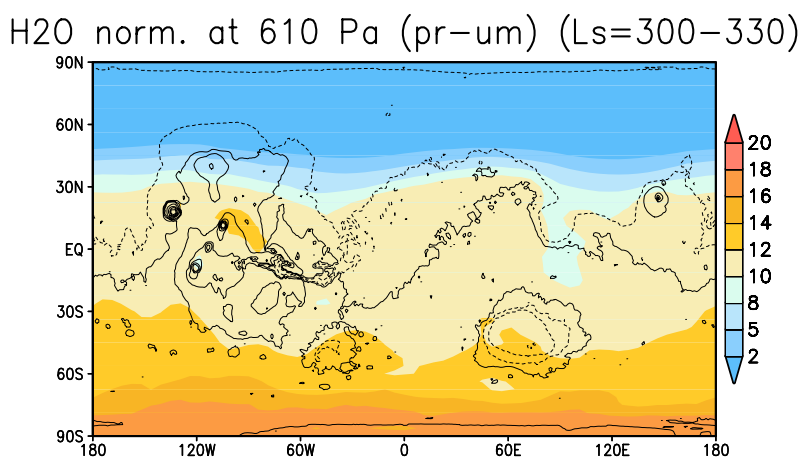

Fig. 7. A GCM map of the water vapor column density, normalized to a pressure of $610 \mathrm{mbar}$, as a function of latitude and longitude, for $L_{\mathrm{s}}=300-330^{\circ}$. Once corrected from the surface pressure, there is no evidence of any longitudinal variations that would be induced by the topography of the Hellas basin.

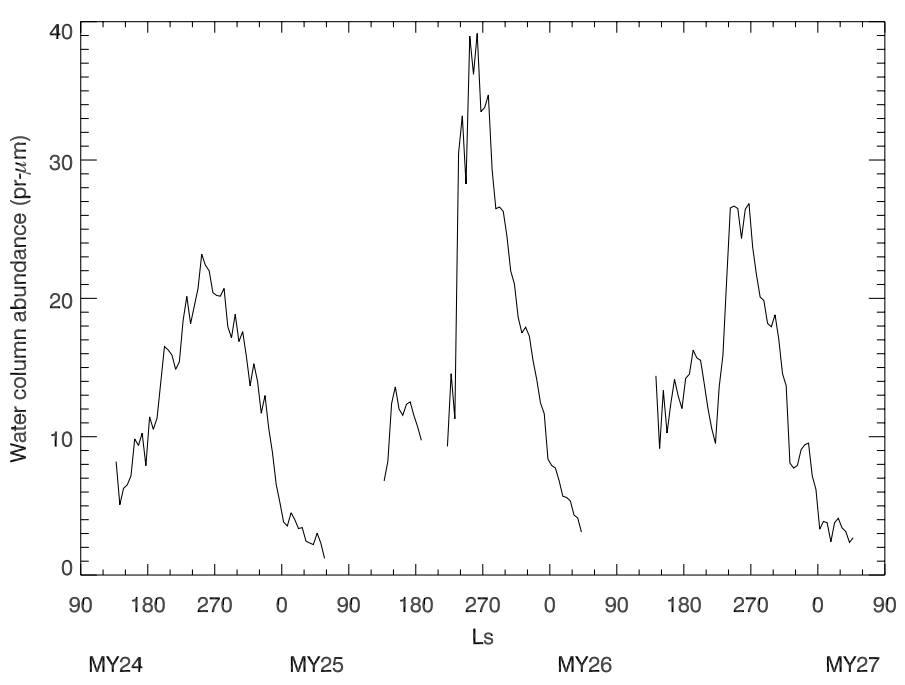

Fig. 8. The water column density over Hellas as a function of solar longitude for MY 24, 25, and 26, extracted from the revised TES database (Smith 2004; smith, priv. comm.; Fouchet et al. 2007).

that most of our data were taken in the afternoon; in particular, the two consecutive Hellas orbits 1935 and 1946, which show very different water contents, both correspond to a local time of 17:00. Also we note that water vapor is undetectable on Orbits 521 and 554, which correspond to early morning (9:00 and 8:00, respectively), but also on Orbit 1186 for which the local time is 14:00. In conclusion, there is no evidence of any correlation between the local time and the water-vapor content over the Hellas basin.

\subsection{Comparison with other observations}

Our data can be compared with the TES results (Smith 2004), obtained over previous martian years. Figure 9 shows the watervapor column density retrieved from the revised TES database (Smith, priv. comm.; Fouchet et al. 2007) over Hellas for martian years 24, 25, and 26. The Mars Express observations started at the end of MY 26 so there is very little overlap, but the general trend of the water vapor variation, as a function of $L_{\mathrm{s}}$, is reproduced over the seasons. The low value of the $\mathrm{H}_{2} \mathrm{O}$ column density (less than $5 \mathrm{pr}-\mu \mathrm{m})$ around the equinox $\left(L_{\mathrm{s}}=330-60^{\circ}\right)$ and the maximum value around the southern solstice $\left(L_{\mathrm{s}}=270^{\circ}\right)$ generally agree with our results. We note, however, that there are strong variations in the TES maximum value between two

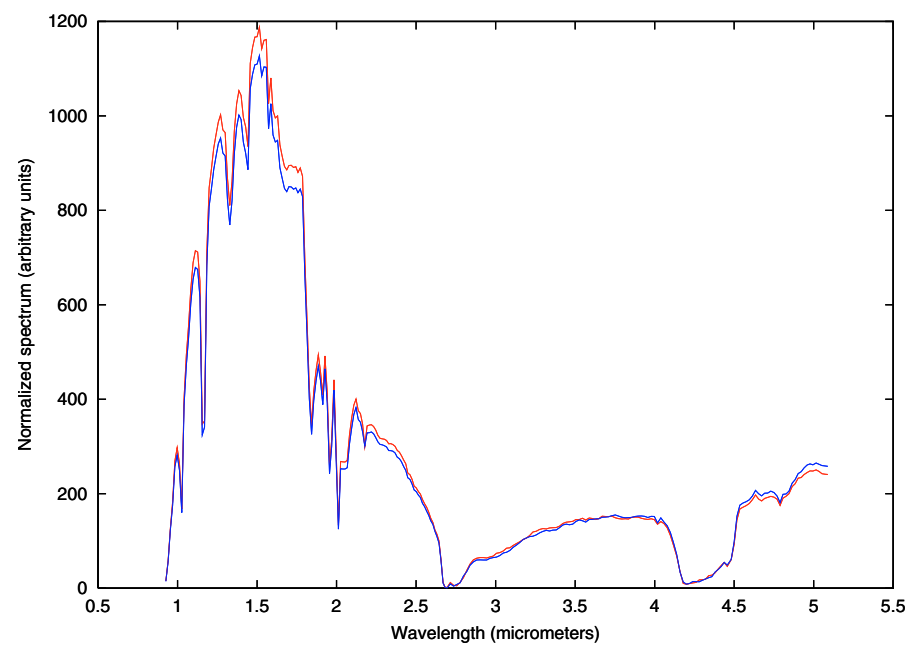

Fig. 9. The averaged normalized OMEGA spectrum over Hellas for Orbit 1935 (red curve) and Orbit 1946 (blue curve), from 1.0 to $5.2 \mu \mathrm{m}$. No significant difference is observed in the albedo, the surface temperature, or the atmospheric temperature.

subsequent martian years (about $25 \mathrm{pr}-\mu \mathrm{m}$ for MY 24 and 26, and almost $40 \mathrm{pr}-\mu \mathrm{m}$ for MY 25). There is no OMEGA or PFS measurement at the season of the expected maximum $\left(L_{\mathrm{s}}=\right.$ $270^{\circ}$ ), but the OMEGA values for $L_{\mathrm{s}}=254$ and $296^{\circ}$ (MY 27) are consistent with the TES data of the previous year. It should also be mentioned that the sharp increase in the water vapor content, observed by OMEGA around $252^{\circ}$, is also observed in the MY 25 and 26 TES data.

One could wonder whether these abrupt and irregular variations might be the onset of local dust storms, frequent during southern spring and summer. To investigate this possibility, we looked for possible variations in the albedo, the surface temperature and the atmospheric temperature in the Hellas basin between Orbits 1935 and 1946. However, the OMEGA images recorded in the visible range do not show any evidence of a change. In addition, the full OMEGA spectra, with both the solar reflected and thermal components, show no significant change in albedo or in the surface temperature (Fig. 9). The albedo in Orbit 1935 is higher and the thermal flux lower by a few percent, which corresponds to a change in the surface temperature by less than $2 \mathrm{~K}$. In addition, the thermal atmospheric profiles are exactly the same on both orbits, as illustrated by the shapes of the wings of the $4.2-\mu \mathrm{m} \mathrm{CO}_{2}$ band. We also looked for possible local variations in the water vapor content within Hellas in Orbit 1946, but the depth of the $\mathrm{H}_{2} \mathrm{O}$ band shows no variation over the whole sequence. Finally, we checked that the water vapor increase observed between Orbits 1935 and 1946 is limited to the Hellas basin, by looking at spectra south of Hellas on both orbits, as illustrated in Fig. 5. Spectra taken south of Hellas (between $50 \mathrm{~S}$ and $64 \mathrm{~S}$ latitudes) show a similar $\mathrm{H}_{2} \mathrm{O}$ band; the relatively large depth of the band stems from the increased airmass during this session, combined to the higher water vapor content toward southern latitudes, as expected by the GCM (Fig. 7). The origin of the sharp water vapor increase is thus still an open question.

Finally, it can be mentioned that another analysis of the water vapor over Hellas is being performed by Maltagliati et al. (2007) from OMEGA data, using the method developed by the authors for the study of $\mathrm{H}_{2} \mathrm{O}$ over the volcanoes (Maltagliati et al. 2008). Their results are in full agreement with the present study. 
In conclusion, the Mars Express data allow us to extend the TES water vapor database over Hellas beyond the TES lifetime, by using a different retrieval method. The OMEGA and PFS results are consistent with the revised TES database. With respect to the GCM calculations, we find good agreement around the southern summer and winter solstices, but a strong discrepancy around the northern spring equinox, for which the observed water vapor abundances are very low. The same discrepancy appears from the comparison of the TES data with the GCM. Further improvements in the GCM calculations are in progress to fit the observations better.

Acknowledgements. We are grateful to M. D. Smith for helpful comments regarding this paper.

\section{References}

Bibring, J.-P., Soufflot, A., Berthé, M., et al. 2004, ESA SP-1240, 37 Conrath, B. J., Curran, R., Hanel, R., et al. 1973, J. Geophys. Res., 78, 4267

Encrenaz, T., Melchiorri, R., Fouchet, T., et al. 2005, A\&A, 441, L9

Encrenaz, T., Fouchet, T., Melchiorri, R., et al. 2006, A\&A, 459, 265

Farmer, C. B., Davis, D. W., Holland, A. L., et al. 1977, J. Geophys. Res., 82, 4225

Fedorova, A. A., Rodin, A. V., \& Baklanova, I. V. 2004, Icarus, 171, 54

Fedorova, A. A., Korablev, O., Bertaux, J.-L., et al. 2007, BAAS, 39, 31.03
Forget, F., Hourdin, F., Fournier, R., et al. 1999, J. Geophys. Res., 104, 24155 Forget, F., Montabone, L., \& Lebonnois, S. 2006, Second International Workshop on Mars Atmosphere Modelling and Observations, Granada, Feb. 27-Mar. 3, 2006, Abstract 4.2.2

Formisano, V., Angrilli, F., Arnold, G., et al. 2005, Plan. Space Sci., 53, 963

Fouchet, T., Lellouch, E., Ignatiev, N., et al. 2007, Icarus, 190, 32

Gamache, R. R., Neshyba, S. P., Plateaux, J. J., et al. 1995, J. Mol. Spectrosc., 170,131

Giuranna, M., Formisano, V., Biondi, D., et al. 2005., Planet. Space Sci., 53, 993 Jacquinet-Husson, N., Arie, E., Ballard, J., et al. 1999, J. Quant. Spectr. Rad. Trans., 62, 205

Jakosky, B. M., \& Haberle, R. M. 1992, in Mars, ed. H. H. Kieffer, et al. (University of Arizona Press)

Lewis, S. R., Collins, M., Read, P. L., et al. 1999, J. Geophys. Res., 104, 24177

Maltagliati, L., Titov, D., Keller, H. U., et al. 2006, Second workshop on Mars atmosphere modelling and observations, ed. F. Forget, et al., Granada

Maltagliati, L., Titov, D., Encrenaz, T., et al. 2007, Proc. of the European Mars Science and Exploration Conf., Noordwijk, 262

Maltagliati, L., Titov, D., Encrenaz, T., et al. 2008, Icarus, 194, 53

Melchiorri, R., Encrenaz, T., Fouchet, T., et al. 2007a, Plan. Space Sci., 55, 333

Melchiorri, R., Encrenaz, T., Drossart, P., et al. 2007b, BAAS, 39, 31.04

Smith, M. D. 2002, J. Geophys. Res., 107, E11, 5115

doi: $10.1029 / 2001 / J E 001522$

Smith, M. D. 2004, Icarus, 167, 148

Sprague, A. L., Hunten, D. M., Hill, R. E., et al. 1996, J. Geophys. Res., 101 23229

Sprague, A. L., Boynton, W. V., Kerry, K. E., et al. 2004, Science, 306, 1364 